\title{
Presencia Bilateral del Canal Incisivo de la Mandíbula. Una Estructura Anatómica con Relevancia Clínica
}

\author{
Bilateral Presence of Mandibular Incisive Canal. An Anatomical Structure with Clinical Relevance
}

"Víctor Beltrán; "Mario Cantín; "Ramón Fuentes Fernández \& "*Wilfried Engelke

BELTRÁN, V.; CANTÍN, M.; FUENTES, F. R. \& ENGELKE, W. Presencia bilateral del canal incisivo de la mandíbula. Una estructura anatómica con relevancia clínica. Int. J. Morphol., 29(2):543-549, 2011.

RESUMEN: La región anterior de la mandíbula generalmente se considera un área quirúrgica segura, con pocos riesgos de daño a estructuras anatómicas importantes. Sin embargo, esta región contiene un canal intraóseo, el canal incisivo de la mandíbula (CIM), que es la continuación del canal mandibular mesial al foramen mental, donde se encuentra el nervio incisivo de la mandíbula acompañado de estructuras vasculares. Su presencia y el curso de la inervación mandibular anterior siguen siendo un tema de debate y algunos autores incluso niegan su existencia. Este reporte define el curso anatómico del CIM bilateralmente mediante tomografía computarizada, confirmando la presencia del nervio incisivo de la mandíbula mediante técnica microquirúrgica asistida por endoscopía. El conocimiento de la presencia, longitud y diámetro del CIM pueden jugar un rol importante en el éxito de la oseointegración de un implante y prevenir alteraciones sensoriales postoperatorias.

PALABRAS CLAVE: Canal incisivo de la mandíbula; Nervio incisivo de la mandíbula; Implante dental; Cirugía mandibular.

\section{INTRODUCCIÓN}

La región anterior de la mandíbula, ubicada entre los forámenes mentales, es considera un área óptima para la colocación de implantes dentales, con un mínimo riesgo de daño a estructuras anatómicas importantes en los diversos procedimientos quirúrgicos que son llevados a cabo.

Sin embargo, esta región contiene canales intraóseos con elementos vasculares y ramos nerviosos provenientes directamente del nervio alveolar inferior (NAI) (Gahleitner et al., 2001; Tepper et al., 2001). Olivier (1928) describió por primera vez el trayecto anatómico del NAI, el cual ingresa por el foramen mandibular, recorre el cuerpo de la mandíbula a través del canal mandibular dirigiéndose hasta el foramen mental, donde se divide en dos ramos terminales, el nervio mental (Nervus mentalis) que emerge por el foramen mental y el nervio incisivo, el cual se dirige hacia la zona anterior de la mandíbula (Kqiku et al., 2010), generalmente rodeado por un canal óseo compacto que representa la continuación intraósea medial al foramen mental del canal mandibular, denominado canal incisivo de la mandíbula (CIM) (Mardinger et al., 2000).
Para diversos autores la anatomía precisa y contenido del CIM son controversiales (Denissen et al., 1984; Polland et al., 2001; Haribhakti, 1996; Madeira et al., 1978; Pogrel et al., 1997; Serman, 1989; Mardinger et al.), y en muchos libros y artículos anatómicos aún no existe referencia de su existencia (Makris et al., 2010; Liu et al., 2009; Jacobs et al., 2007). Mediante radiografías dentales intraorales y panorámicas, donde se pueden visualizar estructuras como el foramen mental y el canal mandibular en toda su extensión, muchas veces no es posible observar la presencia del CIM (Lindh et al., 1992; Mraiwa et al., 2003; Jacobs et al., 2004; Uchida et al., 2009). Además, en mandíbulas desdentadas debemos considerar el proceso de reabsorción de tipo centrífuga que sufre esta zona anatómica, inverso al patrón de reabsorción maxilar (Pietrokovski et al., 2007).

Con la creciente utilización de implantes dentales, extracción de bloques óseos mandibulares, colocación de injertos, placas de osteosíntesis, mentoplastías, etc. en la región anterior de la mandíbula (Mardinger et al.), el núme-

\footnotetext{
* Departamento de Odontología Integral, Facultad de Medicina, Universidad de La Frontera, Chile.

** Dentistry Centre, Department of Maxillofacial Surgery, University of Göttingen, Germany.
} 
ro de quejas postoperatorio ha ido en aumento (Jacobs et al., 2007), donde la existencia del CIM ha sido referida por los informes de complicaciones post-quirúrgicas. Se han reportado trastornos de sensibilidad causada por traumatismo directo del CIM tras la instalación de implantes en la región anterior de la mandíbula (Jacobs et al., 2007; Romanos \& Greenstein, 2009). Junto a la alteración neurosensorial, se han descrito complicaciones como hematomas, edema y fracaso de la oseointegración de los implantes durante o después de procedimientos quirúrgicos (Kohavi \& Bar-Ziv, 1996; Rosenquist 1996; von Arx et al., 2005; Romanos \& Greenstein; Makris et al.). En 1996, Kohavi \& Bar-Ziv presentaron un caso de un paciente con fuerte dolor y alteración de la sensibilidad después de la inserción de un implante en el área de los incisivos laterales mandibulares. Tras realizar un exámen de tomografía computarizada (TC), se reveló la presencia de un canal en la parte anterior de la mandíbula, contínuo por inferior a los implantes, en dirección anterior y mediana como una estructura específica e identificada como el CIM y su nervio respectivo.

En consecuencia, para evitar estas complicaciones es necesario reconocer la existencia del CIM como una estructura anatómica normal, así como su localización exacta en cada caso particular, el cual debe ser identificado previo a cualquier procedimiento quirúrgico en el sector anterior de la mandíbula mediante una técnica imagenológica apropiada.

El propósito de este artículo es reportar la presencia bilateral del canal incisivo de la mandíbula y discutir sus características anatómicas e implicancias clínicas y quirúrgicas.

\section{REPORTE DE CASO}

Paciente de 60 años de edad, de sexo femenino, sin compromiso sistémico a la anamnesis médica. En la anamnesis odontológica remota, la paciente relata pérdida total de los dientes mandibulares y maxilares principalmente por enfermedad periodontal, no entregando antecedentes de traumas en la región mandibular. En la anamnesis odontológica actual la paciente presenta una reabsorción avanzada de zona anterior y posterior mandibular, no pudiendo ser portadora de prótesis mandibular por falta de retención (Fig. 1). Este proceso se acompaña de un perfil anterior alterado, disminuido en sentido vertical y aumentado en sentido anteroposterior por una reabsorción de patrón centrífugo, generando un perfil de tipo prognata.

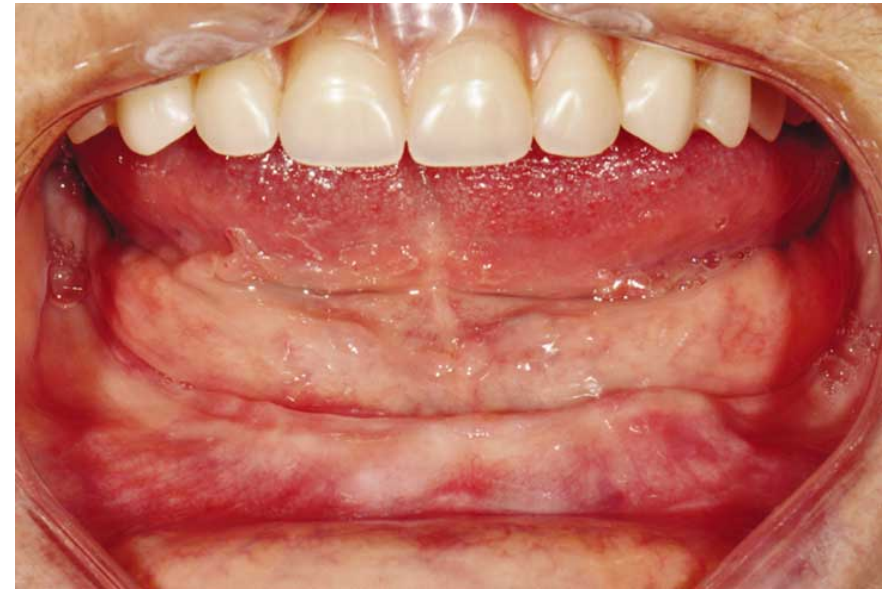

Fig. 1. Visión intraoral del reborde mandibular anterior desdentado con reabsorción ósea avanzada.

Mediante el análisis de TC se realizó la reconstrucción tridimensional de la mandíbula, donde observó la proyección bilateral del canal para el nervio incisivo de la mandíbula extendiéndose 18 milímetros hacia la línea mediana mandibular, con un diámetro menor que el canal mandibular y una trayectoria descendente desde el foramen mental, ascendiendo en su porción terminal, con una dirección antero-posterior paralela a la base de la mandíbula en su porción central (Fig. 2).

Se decidió realizar la inserción de dos implantes endoóseos en la región anterior de la mandíbula para la técnica de sobredentadura en combinación con la técnica de bloques óseos autólogos de posición invertida y fijación con microplacas quirúrgicas. Los implantes fueron planificados bajo imagenología tridimensional y bidimensional por TC, junto a una guía quirúrgica con tubos estratégicos (Figs. 2 y 3A-B).

Se realizó un abordaje mucoperióstico del sector anterior con descarga anterior para facilitar la remoción de los bloques óseos (Fig. 4A) y posteriormente se utilizó endoscopia con soporte rígido y técnica de inmersión endoscópica bilateral para la visualización del lecho óseo expuesto tras la remoción de los bloques mandibulares y facilitar de esta manera la eliminación del nervio en el sitio quirúrgico por fresado bajo visión endoscópica. Se utilizó óptica endoscópica de 1,9 mm de diámetro con $30^{\circ}$ de angulación y fibra óptica con irrigación continua. Las imágenes obtenidas fueron registradas por un procesador de imagen digital (Karl Storz, Tuttlingen, Alemania) obteniendo una óptima visualización del nervio incisivo de la mandíbula en el lecho quirúrgico previo a la colocación del implante endoóseo (Fig. 4B). Finalmente se realizó la colocación de los implantes. La paciente fue controlada continuamente 30 días post-cirugía, sin relatar presencia de alteraciones sensoriales o dolor. 


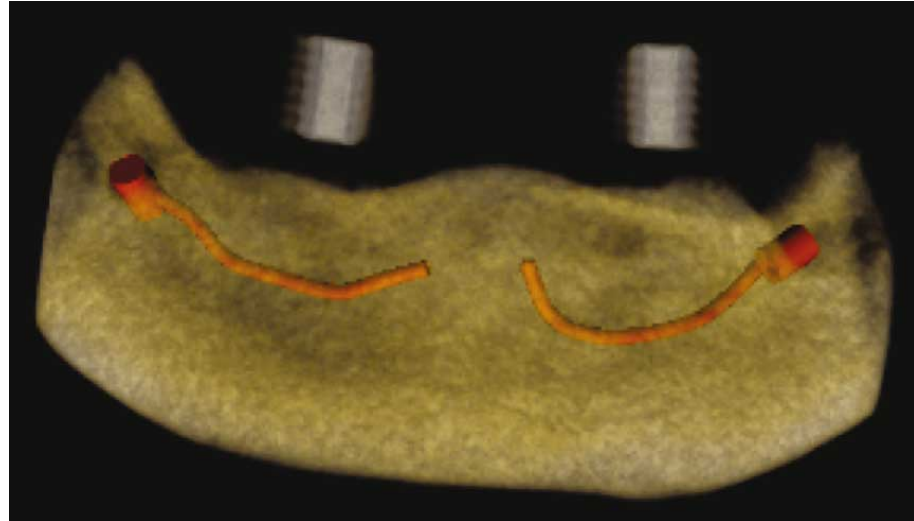

Fig. 2. Visualización del canal mandibular incisivos derecho e izquierdo mediante reconstrucción tridimensional.

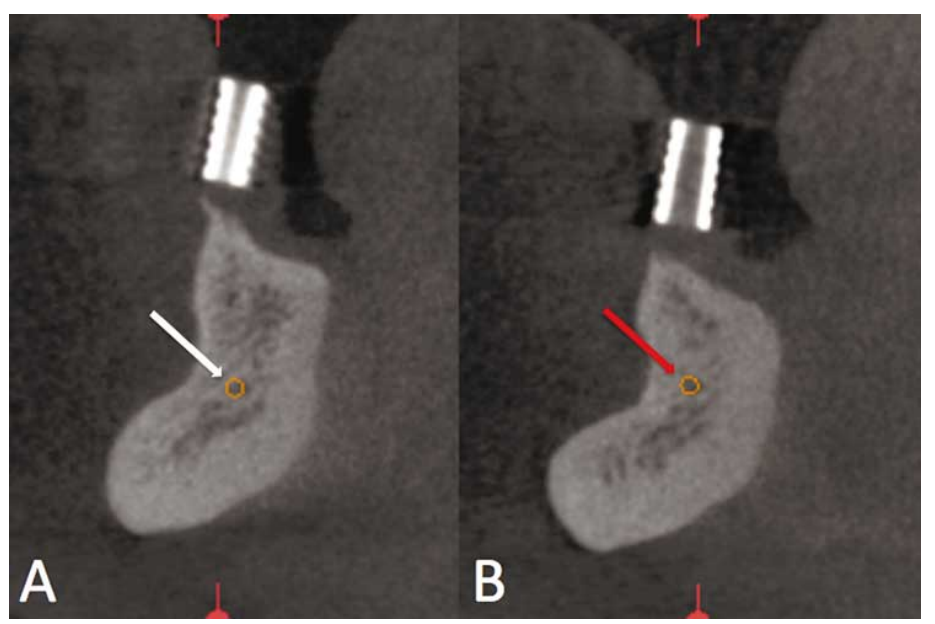

Fig. 3. Corte parasagital mediante TC a nivel del tubo quirúrgico estratégico ubicado en relación a los caninos mandibulares. A. Canal incisivo de la mandíbula derecho (flecha blanca). B. Canal incisivo de la mandíbula izquierdo (flecha roja).

\section{DISCUSIÓN}

Numerosas investigaciones y textos describen detalladamente las estructuras anatómicas de la región posterior de la mandíbula, sin embargo, muy pocos estudios anatómicos, histológicos y radiológicos se han centrado en las estructuras de la región anterior (Madeira et al.; McDonnell et al., 1994; Mardinger et al.; De Andrade et al., 2001; Jacobs et al., 2002, 2004; Mraiwa et al.).

El nervio mental no es el único ramo terminal del NAI (Jacobs et al., 2007). Olivier ya en el año 1928 definió al nervio incisivo de la mandíbula como un ramo del NAI con un curso intraóseo mesial al foramen mental recorriendo el CIM (Mraiwa et al.). Diversos autores han confirmado esta división anatómica del NAI en ramos mental e incisivo (Madeira et al.; Tepper et al., 2001; Lustig et al., 2003), concluyendo que el nervio incisivo y su canal corresponden a una estructura anatómica importante y constante en lugar de una variación anatómica (De Andrade et al.). Se acepta que el origen del nervio incisivo y el CIM se encuentra a nivel del foramen mandibular (Mardinger et al.; Kqiku et al.), sin embago se han descrito algunas variaciones. Wadu et al. (1997) encontraron que el tronco principal del NAI se dividió a nivel de la región molar en los ramos mental e incisivo, los que corrieron paralelos uno del otro dentro del canal y se comunicaron mediante una red de pequeñas fibras. Kqiku et al. estudiaron la

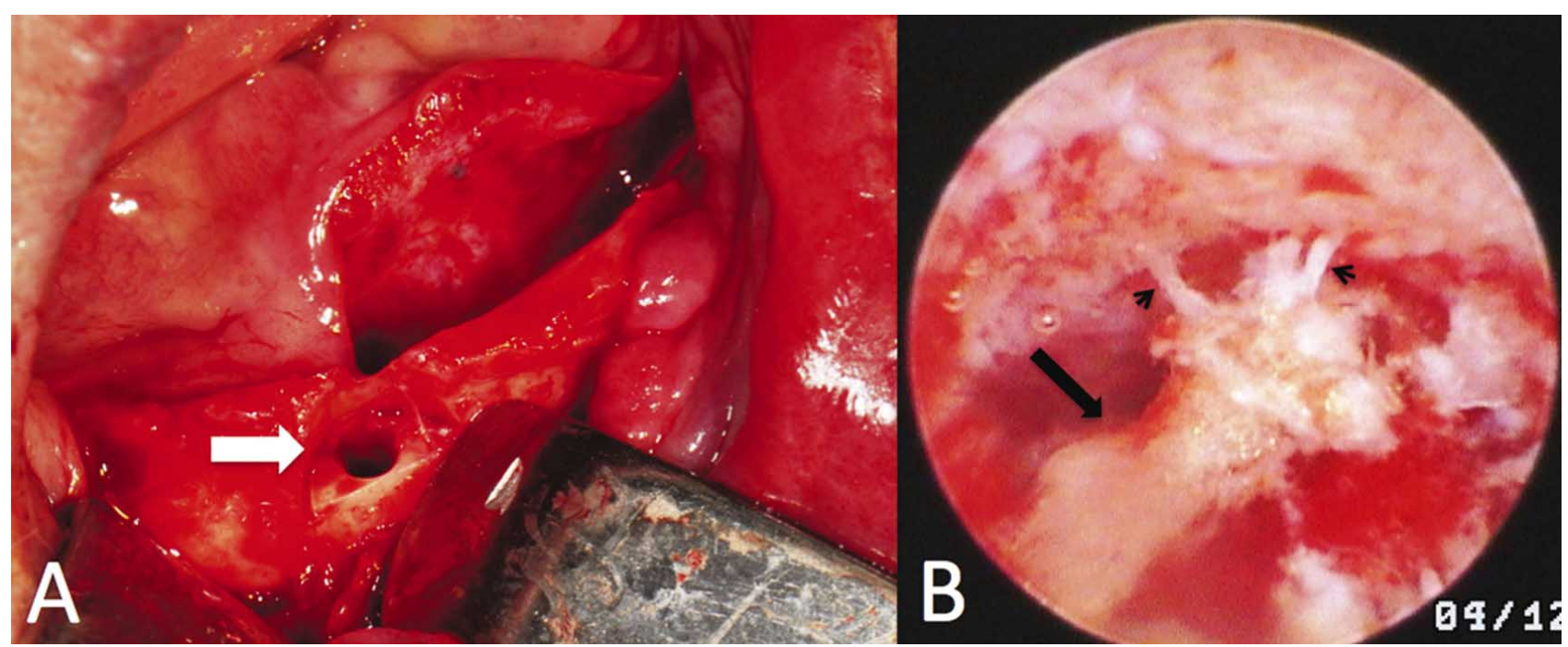

Fig. 4. A. Lecho óseo obtenido tras la remoción del bloque óseo y fresado para la colocación del implante endoóseo en zona mandibular anterior derecha (Flecha). B. Visualización mediante endoscopia de inmersión del nervio incisivo del lado derecho posterior a la remoción del techo del canal por fresado (flecha), desde el cual emergen pequeños ramos nerviosos terminales (cabezas de flecha). 
histología y el curso intraóseo del NAI como un paquete único compuesto de dos nervios que se envuelven en espiral entre sí, y se dividen en la zona molar en el nervio incisivo, que se dirige hacia los caninos e incisivos y, el nervio mental que emerge a través de el foramen mental.

Estudios anatómicos realizados en mandíbulas humanas han demostrado la presencia del CIM con diversas conclusiones. McDonnell et al. evaluaron 331 cadáveres encontrando el CIM en el $99 \%$ de los casos. Mardinger et $a l$ analizaron 46 hemimandíbulas de cadáveres humanos, observando en el total de ellas un elemento nervioso y un paquete vascular saliendo de la mitad inferior del canal mandibular dirigiéndose hacia los incisivos. Estos elementos viajaron a través de una cortical ósea completa en sólo 10 hemimandíbulas, en 27 casos este canal los envolvió parcialmente, en especial en el área mental, encontrando cerca de la línea mediana sólo espacios trabeculares. En 9 hemimandíbulas el nervio viajó completamente a través del espacio trabecular sin pared ósea cortical. En nuestro caso, pudimos encontrar el nervio incisivo dentro del CIM, desde el cual se proyectaron diversos ramos de pequeño calibre que ingresaban a los espacios trabeculares.

Obradovic et al. (1993) examinaron 105 mandíbulas de cadáveres, observando la presencia del CIM claramente definido desde mesial del foramen mental en el $92 \%$ de las mandíbulas dentadas ( $\mathrm{n}=70)$ y sólo en el $31 \%$ de las mandíbulas desdentadas $(\mathrm{n}=30)$. Por otra parte Dennisen et al. no observaron el CIM en mandíbulas atróficas de cadáveres, lo que fue corroborado por Haribhakti. Sin embargo Mraiwa et al. al estudiar 40 mandíbulas edéntulas y 10 parcialmente desdentadas confirmaron la existencia del CIM, el cual fue observado en el $96 \%$ de todas las mandíbulas, cuestionando las observaciones de estudios anteriores, donde probablemente las técnicas utilizadas para localizar esta estructura no poseían la sensibilidad adecuada. Por otro lado, no debemos olvidar el proceso de reabsorción mandibular, el cual, dependiendo principalmente de la cantidad de años transcurridos desde la pérdida dentaria o los factores mecánicos condicionantes como el uso de prótesis dentales, lo que determinará la distancia que pueda existir entre una estructura neurovascular y la cortical alveolar, mientras que en relación con la base esta permanecerá constante (Pietrokovsky et al.).

En general, la alta tasa de incidencia del CIM reportada en los estudios anatómicos hace poco probable que sea considerado como una variación anatómica. De Andrade et al. llegaron a la conclusión que el CIM es una estructura normal, la que se extiende cerca de la línea mediana mandibular, y su nervio incisivo, es la fuente de inervación de los incisivos centrales y laterales, caninos, y en ocasio- nes los primeros premolares mandibulares. Estas conclusiones coinciden con lo encontrado en nuestro caso.

En cuanto a su extensión, según Juodzbalys et al. (2010) el CIM se origina a nivel del foramen mental y termina a nivel del ápice del incisivo lateral o a veces del incisivo central de la mandíbula. De Andrade et al. reportan una longitud promedio de 20,58mm (DE 2,99mm). Nuestros exámenes mostraron una longitud de $18 \mathrm{~mm}$, ubicándonos dentro del promedio métrico reportado por De Andrade et al., pudiendo inferir que esta distancia puede ser tomada como una zona de riesgo para futuras intervenciones.

En relación a su diámetro, Mraiwa et al. reportaron un promedio del CIM de 1,8mm (DE $\pm 0,5)$. Para Mardinger et al. quienes midieron el diámetro del CIM a nivel de origen, a 4 milímetros en dirección mesial, a nivel del canino y a nivel de los incisivos laterales mandibulares, reportaron valores de 2,09mm (DE $\pm 0,42), 1,69 \mathrm{~mm}$ (DE $\pm 1,69), 1,25 \mathrm{~mm}$ $(\mathrm{DE} \pm 0,24)$ y $0,98 \mathrm{~mm}(\mathrm{DE} \pm 0,22)$ respectivamente, demostrando una disminución de su lumen. Esto contrasta con las conclusiones reportadas por Arzouman et al. (1993), para quienes no existe diferencia en el diámetro del CIM en sujetos dentados o desdentados.

La presencia del CIM también ha sido estudiada a través de exámenes imagenológicos. Varios estudios (Mardinger et al.; Tepper et al.; Jacobs et al., 2002, 2004; Mraiwa et al.) se han centrado en la visualización del CIM mediante radiografías convencionales y exámenes de TC. Mardinger et al. mediante radiografías panorámicas determinaron que sólo en el 24\% de los casos la imagen del CIM fue bien evaluada por tener bordes corticales bien definidos, en el $32 \%$ los márgenes radiográficos fueron mal definidos y en el $44 \%$ de los casos el CIM no podía ser visto. Mraiwa et al. reportaron que en radiografía panorámica el CIM fue visualizado como una prolongación del canal mandibular en el 50\% de las mandíbulas investigadas, mientras que Jacobs et al. (2002) informaron que el CIM fue identificado sólo en el 15\% de las 545 radiografías panorámicas, con buena visibilidad en sólo el 1\%. Estos resultados nos permiten concluir que aunque el CIM esta presente, como ha sido descrito mediante los estudios anatómicos, generalmente no es visible con radiografías convencionales.

Estos resultados varian ampliamente al utilizar exámenes imagenológicos más avanzados que permiten una mejor calidad y definición de las estructuras, en donde a través de TC pudimos comprobar la ubicación exacta del CIM en todo su curso, permitiéndonos realizar la correcta planificación de posición para nuestros implantes. Diversos autores han comprobado la utilidad de estos exámenes. Jacobs et al. (2004) examinaron 230 TC en espiral para la 
planificación preoperatoria de implantes en la zona posterior de la mandíbula, donde el CIM se identificó en el 93\% de los casos, con buena visibilidad en el $22 \%$ de los casos. Makris et al. evaluaron la visibilidad y el curso del CIM mediante TC, reportando que fue definitivamente visible en el $83,5 \%$ de las exploraciones, terminando en promedio a $15 \mathrm{~mm}$ por delante del foramen mental. La distancia media desde el borde inferior de la mandíbula fue $11,5 \mathrm{~mm}$ y su curso estuvo más cerca de la cortical vestibular de la mandíbula en el $87 \%$ de las exploraciones. La alta tasa de detección del CIM mediante TC indica el alto valor preoperatorio del análisis para los procedimientos quirúrgicos en el sector anterior mandibular. Estos resultados concuerdan con los reportados por Tepper et al. y Jacobs et al. (2002), donde el CIM fue visible en el $94 \%$ de los casos mediante la TC helicoidal. De Andrade et al. en TC reportaron una longitud del CIM entre 20,58 y 21,45mm, visiblemente mayor a la longitud reportada por los estudios anatómicos.

El reconocimiento relativamente exacto de estas estructuras anatómicas es fundamental para evitar una serie de complicaciones, puesto que el CIM contiene un paquete neurovascular de características similares al paquete neurovascular alveolar inferior, lo que ha sido confirmado recientemente por Kqiku et al. mediante estudios histológicos.

Wismeijer et al. (1997) estudiaron 110 pacientes desdentados que fueron tratados mediante sobredentadura sostenida por dos o cuatro implantes interconectados. Aunque el foramen mental se identificó siempre durante la operación y todos los implantes fueron insertados por lo menos 3 mm medial al borde anterior del foramen mental, la alteración sensorial permanente en el labio inferior se observó en el $7 \%$ de los casos. Esta alteración sensorial está relacionada con el daño del CIM y su nervio, así como también con la presencia de dolor intra y post-quirúrgico (Kohavi \& BarZiv).

Pueden haber varias explicaciones para estos fenómenos. En primer lugar el trauma directo al paquete neurovascular incisivo de la mandíbula (Kohavi \& Bar-Ziv; Mardinger et al.; von Arx et al.; Makris et al.) que puede resultar en daño nervioso directo por el fresado o bien una neuropraxia de éste (Raghoebar et al., 2007). En segundo lugar, un traumatismo en el CIM puede causar un hematoma que acturía con efecto de cámara cerrada y se ramificaría hacia el nervio incisivo, nervio mental, inclusive haste el NAI, ya el hematoma produciría presión resultando en daño axonal, un traumatismo indirecto (Mraiwa et al.; Jacobs et al., 2007). El tercero se refiere a la posible presencia de un bucle del nervio mental extendiéndose hacia anterior (Mardinger et al.). Romano \& Greenstein mencionan inclu- so la posibilidad de que un daño en el CIM podría causar la formación de un neuroma traumático. No obstante, otra posibilidad tiene que ver con factores físicos o mecánicos inherentes al fresado óseo o irrigación insuficiente sobre una estructura ósea eminentemente cortical y poco vascularizada generando necrosis ósea.

Otra complicación reportada por la perforación de un CIM es el fracaso de la oseointegración de los implantes, debido a la migración apical del tejido blando alrededor del implante (Rosenquist). Complicación similar se ha reportado cuando implantes se han colocado contiguos al canal incisivo del maxilar (Makris et al.).

Según De Andrade et al. el CIM y su nervio deben ser tomados en cuenta al utilizar la zona del mentón y sínfisis mandibular como fuente de hueso autógeno para injertos o en caso de mentoplastías con el fin de reducir la morbilidad de daño neurovascular postoperatorio. Un estudio retrospectivo realizado por von Arx et al. reportó un 6\% de alteraciones sensoriales después de la extracción del hueso del menton, la que aumentó exponencialmente con la cantidad de hueso esponjoso eliminado, asumiendo el daño del CIM y la lesión su nervio incisivo como factor causal. La pérdida de sensibilidad pulpar también ha sido reportada después de la cirugía de extracción de hueso mandibular hasta en un $80 \%$ de los pacientes, siendo persistente en el $20 \%$ de los casos (Pommer et al.). Según Raghoebar et al. la morbilidad del sitio donante implica el sangrado intraoperatorio, dehiscencia de la herida, lesión del nervio incisivo, obliteración del canal pulpar, así como la pérdida de la sensibilidad de la pulpa de los dientes anteroinferiores.

Debemos considerar a la región anterior de la mandíbula, como una zona anatómica principalmente de hueso compacto, sujeta a errores diagnósticos de la imagenología convencional para la localización de estructuras neurovasculares, donde la aproximación imagenológica tridemensional nos ha permitido un diagnóstico pre-quirúrgico más preciso (Jacobs et al., 2007; Pommer et al., 2008; Uchida et al.). No obstante, nuevas tecnologías como la endoscopía están aún en desarrollo y prometen aproximaciones a la microcirugía de esta zona anatómica (Engelke, 2002). Además, es fundamental tomar en cuenta las diferencias que presentarán las mandíbulas desdentadas, donde el mayor cambio ocurre a nivel del proceso alveolar que sufre una reabsorción ósea extensa y progresiva, siendo el grado de reabsorción de tipo centrífuga después de la extracción del diente más variable en la región anterior (Polland et al.).

Finalmente, si bien nos hemos referido a la presencia del CIM, siendo avalada por nuestros exámenes y la lite- 
ratura científica actual, aún no existe una referencia clara por los textos oficiales ni una denominación específica por la terminología anatómica internacional, tanto en su raíz del latín como en la mayoría de los vernáculos, donde el término de referencia canal incisivo de la mandíbula propuesto implica un valor descriptivo adecuado, el cual relaciona sus característica morfológicas y funcionales.

En conclusión, la presencia y anatomía intraósea del CIM y su nervio no deben ser ignoradas en la planificación de implantes en la zona anterior, procedimientos de recolección hueso del mentón, cirugía ortognática, incluso en la cirugía periapical. Esto podría jugar un rol fundamental para evitar todo riesgo de daño neurovascular y obtener la osteointegración exitosa de un implante. Sin embargo, la habilidad del clínico para definir este dato a partir de radiografías convencionales es limitada, por lo que la TC se recomienda para una lograr una visualización más exacta de las estructuras anatómicas que deben ser identificadas previamente a cualquier procedimiento quirúrgico en esta región. Las nuevas tecnologías como la endoscopía con soportes rígidos en combinación con la localización imagenológica tridemensional, nos ofrecen una concepción microquirúrgica para un abordaje exitoso.

BELTRÁN, V.; CANTÍN, M.; FUENTES, F. R. \& ENGELKE, W. Bilateral presence of mandibular incisive canal. An anatomical structure with clinical relevance. Int. J. Morphol., 29(2):543-549, 2011.

SUMMARY: The anterior mandible region is generally considered a safe surgical area, involving few risks of damage to vital anatomic structures. However, this area has an intraosseous canal, the mandibular incisive canal (MIC), the continuation of the mandibular canal, mesial to the mental foramen where the mandibular incisive nerve along with vascular structures is located. The presence and course of the incisive nerve remains a matter of debate and some authors even neglect it. This case report defines the anatomic courses of the incisive mandibular canal bilaterally using cone-beam computed tomography and confirms the presence of the incisive nerve by endoscopically assisted surgical technique. The knowledge of the MIC presence, length and diameter may play an important role in successful implant osseointegration and prevention of postoperative sensory disturbances.

KEY WORDS: Mandibular incisive canal; Mandibular incisive nerve; Dental implant; Mandibular surgery.

\section{REFERENCIAS BIBLIOGRÁFICAS}

Arzouman, M. J.; Otis, L.; Kipnis, V. \& Levine, D. Observations of the anterior loop of the inferior alveolar canal. Int. J. Oral Maxillofac. Implants, 8(3):295-300, 1993.

De Andrade, E.; Otomo-Corgel, J.; Pucher, J.; Ranganath, K. A. $\&$ St George, N. Jr. The intraosseous course of the mandibular incisive nerve in the mandibular symphysis. Int. J. Periodontics Restorative Dent., 21(6):591-7, 2001.

Denissen, H. W.; Veldhuis, H. A. \& van Faassen, F. Implant placement in the atrophic mandible: an anatomic study. $J$. Prosthet. Dent., 52(2):260-3, 1984.

Engelke, W. G. In situ examination of implant sites with support immersion endoscopy. Int. J. Oral Maxillofac. Implants, 17(5):703-6, 2002.

Gahleitner, A.; Hofschneider, U.; Tepper, G.; Pretterklieber, M.; Schick, S.; Zauza, K. \& Watzek, G. Lingual vascular canals of the mandible: evaluation with dental CT. Radiology, 220(1):186-9, 2001.

Haribhakti, V. V. The dentate adult human mandible: an anatomic basis for surgical decision making. Plast. Reconstr. Surg., 97(3):536-41; discussion 542-3, 1996.
Jacobs, R.; Mraiwa, N.; van Steenberghe, D.; Gijbels, F. \& Quirynen, M. Appearance, location, course, and morphology of the mandibular incisive canal: an assessment on spiral CT scan. Dentomaxillofac. Radiol., 31(5):322-7, 2002.

Jacobs, R.; Mraiwa, N.; van Steenberghe, D.; Sanderink, G. \& Quirynen, M. Appearance of the mandibular incisive canal on panoramic radiographs. Surg. Radiol. Anat., 26(4):32933, 2004.

Jacobs, R.; Lambrichts, I.; Liang, X.; Martens, W.; Mraiwa, N.; Adriaensens, P. \& Gelan, J. Neurovascularization of the anterior jaw bones revisited using high-resolution magnetic resonance imaging. Oral Surg. Oral Med. Oral Pathol. Oral Radiol. Endod., 103(5):683-93, 2007.

Juodzbalys, G.; Wang, H. L. \& Sabalys, G. Anatomy of Mandibular Vital Structures. Part II: Mandibular Incisive Canal, Mental Foramen and Associated Neurovascular Bundles in Relation with Dental Implantology. J. Oral Maxillofac. Res., 1(1):e3, 2010.

Kohavi, D. \& Bar-Ziv, J. Atypical incisive nerve: clinical report. Implant. Dent., 5(4):281-3, 1996.

Kqiku, L.; Weiglein, A. H.; Pertl, C.; Biblekaj, R. \& Städtler, P. Histology and intramandibular course of the inferior alveolar nerve. Clin. Oral Investig., DOI 10.1007/s00784-010-0459$\mathrm{x}, 2010$. 
Lindh, C.; Petersson, A. \& Klinge, B. Visualisation of the mandibular canal by different radiographic techniques. Clin. Oral Implants Res., 3(2):90-7, 1992.

Liu, T.; Xia, B. \& Gu, Z. Inferior alveolar canal course: a radiographic study. Clin. Oral Implants Res., 20(11):1212-8, 2009.

Lustig, J. P.; London, D.; Dor, B. L. \& Yanko, R. Ultrasound identification and quantitative measurement of blood supply to the anterior part of the mandible. Oral Surg. Oral Med. Oral Pathol. Oral Radiol. Endod., 96(5):625-9, 2003.

McDonnell, D.; Reza Nouri, M. \& Todd, M. E. The mandibular lingual foramen: a consistent arterial foramen in the middle of the mandible. J. Anat., 184(Pt2):363-9, 1994.

Madeira, M. C.; Percinoto, C. \& das Graças, M. Silva M. Clinical significance of supplementary innervation of the lower incisor teeth: a dissection study of the mylohyoid nerve. Oral Surg. Oral Med. Oral Pathol., 46(5):608-14, 1978.

Makris, N.; Stamatakis, H.; Syriopoulos, K.; Tsiklakis, K. \& van der Stelt, P. F. Evaluation of the visibility and the course of the mandibular incisive canal and the lingual foramen using cone-beam computed tomography. Clin. Oral Implants Res., 21(7):766-71, 2010.

Mardinger, O.; Chaushu, G.; Arensburg, B.; Taicher, S. \& Kaffe, I. Anatomic and radiologic course of the mandibular incisive canal. Surg. Radiol. Anat., 22(3-4):157-61, 2000.

Mraiwa, N.; Jacobs, R.; Moerman, P.; Lambrichts, I.; van Steenberghe, D. \& Quirynen, M. Presence and course of the incisive canal in the human mandibular interforaminal region: two-dimensional imaging versus anatomical observations. Surg. Radiol. Anat., 25(5-6):416-23, 2003.

Obradovic, O.; Todorovic, L.; Pesic, V.; Pejkovic, B. \& Vitanovic, V. Morphometric analysis of mandibular canal: clinical aspects. Bull. Group Int. Rech. Sci. Stomatol. Odontol., 36(34):109-13, 1993.

Olivier, E. The inferior dental canal and its nerve in the adult. $B r$. Dent. J., 49:356-8, 1928.

Pietrokovski, J.; Starinsky, R.; Arensburg, B. \& Kaffe, I. Morphologic characteristics of bony edentulous jaws. $J$. Prosthodont., 16(2):141-7, 2007.

Pogrel, M. A.; Smith, R. \& Ahani, R. Innervation of the mandibular incisors by the mental nerve. J. Oral Maxillofac. Surg., 55(9):961-3, 1997.

Polland, K. E.; Munro, S.; Reford, G.; Lockhart, A.; Logan, G.; Brocklebank, L. \& McDonald, S. W. The mandibular canal of the edentulous jaw. Clin. Anat., 14(6):445-52, 2001.
Pommer, B.; Tepper, G.; Gahleitner, A.; Zechner, W. \& Watzek, G. New safety margins for chin bone harvesting based on the course of the mandibular incisive canal in CT. Clin. Oral Implants Res., 19(12):1312-6, 2008.

Raghoebar, G. M.; Meijndert, L.; Kalk, W. W. \& Vissink, A. Morbidity of mandibular bone harvesting: a comparative study. Int. J. Oral Maxillofac. Implants, 22(3):359-65, 2007.

Romanos, G. E. \& Greenstein, G. The incisive canal. Considerations during implant placement: case report and literature review. Int. J. Oral Maxillofac. Implants, 24(4):740$5,2009$.

Rosenquist, B. Is there an anterior loop of the inferior alveolar nerve? Int. J. Periodontics Restorative Dent., 16(1):40-5, 1996.

Serman, N. J. The mandibular incisive foramen. J. Anat., 167:1958, 1989.

Tepper, G.; Hofschneider, U. B.; Gahleitner, A. \& Ulm, C. Computed tomographic diagnosis and localization of bone canals in the mandibular interforaminal region for prevention of bleeding complications during implant surgery. Int. J. Oral Maxillofac. Implants, 16(1):68-72, 2001.

Uchida, Y.; Noguchi, N.; Goto, M.; Yamashita, Y.; Hanihara, T.; Takamori, H.; Sato, I.; Kawai, T. \& Yosue, T. Measurement of anterior loop length for the mandibular canal and diameter of the mandibular incisive canal to avoid nerve damage when installing endosseous implants in the interforaminal region: a second attempt introducing cone beam computed tomography. J. Oral Maxillofac. Surg., 67(4):744-50, 2009.

von Arx, T.; Häfliger, J. \& Chappuis, V. Neurosensory disturbances following bone harvesting in the symphysis: a prospective clinical study. Clin. Oral Implants Res., 16(4):432-9, 2005.

Wadu, S. G.; Penhall, B. \& Townsend, G. C. Morphological variability of the human inferior alveolar nerve. Clin. Anat., 10(2):82-7, 1997.

Wismeijer, D.; van Waas, M. A.; Vermeeren, J. I. \& Kalk, W. Patients' perception of sensory disturbances of the mental nerve before and after implant surgery: a prospective study of 110 patients. Br. J. Oral Maxillofac. Surg., 35(4):254-9, 1997.

\section{Dirección para correspondencia: \\ Dr. Víctor Javier Beltrán Varas \\ Dpto. Odontología Integral, Facultad de Medicina \\ Universidad de La Frontera \\ Calle Claro Solar No 115 - Of. 420, Temuco \\ CHILE}

Email: vbeltran@ufro.cl

Recibido : 08-01-2011 Aprobado: 29-02-2011 